\title{
UNCOLOURING OF LIE COLOUR ALGEBRAS
}

\author{
D.S. McAnally and A.J. Brakcen
}

\begin{abstract}
The link between a Lie colour algebra and a corresponding Lie superalgebra is clarified in the case of the general linear algebras. The Lie superalgebra inherits from the colour cocommutative coproduct of the corresponding Lie colour algebra, a super coproduct which differs from the usual one, and is not supercocommutative. It is associated with a new $R$-matrix satisfying the super Yang-Baxter equation.
\end{abstract}

\section{INTRODUCTION}

The recent high level of interest in quantised universal enveloping algebras (quantum groups and supergroups) has placed Lie algebras and superalgebras themselves in a new perspective. They can now be viewed as being associated with a particular limit $(q \rightarrow 1)$ of quantum groups and quantum supergroups, which are more general structures, being quasitriangular Hopf algebras.

One of the principal features of interest of such a Hopf algebra is the existence of a universal element ( $R$-matrix) which intertwines two associated coproducts of the algebra, and provides a solution of the quantum Yang-Baxter equation, and thus may be of interest in the construction of exactly solvable models, for example, in statistical mechanics [1].

The introduction of Lie colour algebras by Rittenberg and Wyler [12] was a promising generalisation of the super case, with the idea that there might be possible applications in particle physics [2, 3, 4], but, although there are to date no important physical applications, colour algebras and Lie colour algebras are interesting and potentially important mathematical structures.

The works of Scheunert $[13,14,15]$ and Kleeman $[7,8,9]$ have established a surprising link between Lie colour algebras and Lie superalgebras, with a well-defined correspondence mapping any Lie colour algebra to a Lie superalgebra, involving however the introduction of new generators from outside the Lie colour algebra. This correspondence between a Lie colour algebra and a Lie superalgebra can be extended to their graded modules $[7,8,9,13,14,15]$ : for each colour-graded module of the

Received 13th June, 1996.

The authors acknowledge the support of a Small Grant from the Australian Research Council.

Copyright Clearance Centre, Inc. Serial-fee code: 0004-9729/97 \$A2.00+0.00. 
Lie colour algebra, there is a corresponding $\mathbb{Z}_{2}$-graded module of the corresponding Lie superalgebra.

Work on quantum supergroups leads naturally to the question of quantisation of colour algebras, in the hope of obtaining new solutions, both of the colour Yang-Baxter equation, and of the non-graded Yang-Baxter equation, leading perhaps to new solvable models. This is the primary motivation for our investigation of Lie colour algebras and their quantisation.

The first step, taken here, will be a re-examination in some detail of the case of the classical Lie colour algebra, to clarify similarities and differences between the colour and super cases.

The results obtained demonstrate a correspondence between each Lie colour algebra of the general linear type and a corresponding Lie superalgebra, in terms of the generators of the Lie colour algebra, without the necessity to introduce generators external to the Lie colour algebra, as in earlier work [7, 8, 9, 13, 14, 15]. Furthermore, a Hopf superalgebra structure can be imposed on the "completed" universal enveloping algebra of the Lie colour algebra, and this Hopf superalgebra structure relates to the natural colour coproduct on the Lie colour algebra. Bringing these two results together affords a method for determining a new (and non-supercocommutative) coproduct structure on the universal enveloping algebra of the Lie superalgebra $g l(m \mid n)$, thus endowing this universal enveloping algebra with a new non-supercocommutative Hopf superalgebra structure and a new, nontrivial, $R$-matrix. This procedure opens the door to the construction of new and interesting solutions of the graded Yang-Baxter equation, when extended to the quantised case, as we shall show in a subsequent article.

\section{Phase functions}

Here we review the basic definitions of phase groups and phase functions, and their properties.

Colour structures over a field $\mathbb{F}$ are a generalisation of superstructures in that the grading is generalised; whereas superstructures are graded with respect to $\mathbb{Z}_{2}$, colour structures are graded with respect to an arbitrary Abelian (additive) group $\Gamma$. The grading is determined by $\Gamma$ and a phase function $\omega: \Gamma \times \Gamma \rightarrow \mathbb{F}$ satisfying

$$
\omega(\alpha, \beta) \omega(\beta, \alpha)=1, \quad \omega(\alpha, \beta+\gamma)=\omega(\alpha, \beta) \omega(\alpha, \gamma)
$$

EXAMPLES. (1) $\Gamma_{S}=\mathbb{Z}_{2}$ and $\omega_{S}(\alpha, \beta)=(-1)^{\alpha \beta}$. This is the now-familiar superstructure case.

(2) $\Gamma_{Q}=\mathbb{Z}_{2} \times \mathbb{Z}_{2}$ and $\omega_{Q}(\alpha, \beta)=(-1)^{\alpha_{1} \beta_{2}+\alpha_{2} \beta_{1}}$, where $\alpha=\left(\alpha_{1}, \alpha_{2}\right), \beta=$ 
$\left(\beta_{1}, \beta_{2}\right) \in \mathbb{Z}_{2} \times \mathbb{Z}_{2}$, so that

$$
\omega_{Q}(\alpha, \beta)=\left\{\begin{array}{l}
-1, \alpha \neq \beta \neq(0,0) \neq \alpha \\
1, \text { otherwise }
\end{array}\right.
$$

that is, $\omega_{Q}(\alpha, \beta)=1$ if and only if either $\alpha$ or $\beta$ equals $(0,0)$ or they are equal.

(3) $\Gamma_{S Q}=\Gamma_{S} \times \Gamma_{Q}=\mathbb{Z}_{2} \times \mathbb{Z}_{2} \times \mathbb{Z}_{2}$ and

$$
\omega_{S Q}(\alpha, \beta)=\omega_{S}\left(\alpha_{1}, \beta_{1}\right) \omega_{Q}\left(\left(\alpha_{2}, \alpha_{3}\right),\left(\beta_{2}, \beta_{3}\right)\right)=(-1)^{\alpha_{1} \beta_{1}+\alpha_{2} \beta_{3}+\alpha_{3} \beta_{2}} .
$$

Note that $\left(\Gamma_{S Q}, \omega_{S Q}\right) \cong\left(\Gamma_{S}, \omega_{S}\right) \times\left(\Gamma_{S}, \omega_{S}\right) \times\left(\Gamma_{S}, \omega_{S}\right)$.

(4) For $n \geqslant 1, \Gamma_{C, n}=\mathbb{Z}_{2}^{\times n}=\mathbb{Z}_{2} \times \cdots \times \mathbb{Z}_{2}$ and

$$
\begin{aligned}
\omega_{C, n}\left(\left(\alpha_{1}, \ldots, \alpha_{n}\right),\left(\beta_{1}, \ldots, \beta_{n}\right)\right) & =(-1)^{\sum_{i \neq j} \alpha_{i} \beta_{j}} \\
& =(-1)^{\left(\sum_{i} \alpha_{i}\right)\left(\sum_{j} \beta_{j}\right)+\sum_{i} \alpha_{i} \beta_{i}}
\end{aligned}
$$

Note that $\left(\Gamma_{C, 2}, \omega_{C, 2}\right)=\left(\Gamma_{Q}, \omega_{Q}\right)$.

(5) $\Gamma_{n}=\mathbb{Z}_{n} \times \mathbb{Z}_{n}$ and $\omega_{n}\left(\left(\alpha_{1}, \alpha_{2}\right),\left(\beta_{1}, \beta_{2}\right)\right)=\zeta_{n}^{\alpha_{1} \beta_{2}-\alpha_{2} \beta_{1}}$ for $\alpha_{1}, \alpha_{2}, \beta_{1}, \beta_{2} \in \mathbb{Z}_{n}$, where $\zeta_{n}=\exp (2 \pi i / n)$. Note that $\left(\Gamma_{2}, \omega_{2}\right)=\left(\Gamma_{Q}, \omega_{Q}\right)$.

(6) For $t \in \mathbb{F}$ nonzero, $\Gamma_{t}^{ \pm \pm}=\mathbb{Z} \times \mathbb{Z}$ and $\omega_{t}^{ \pm \pm}((a, b),(c, d))=( \pm 1)^{a c}( \pm 1)^{b d} t^{a d-b c}$ for $a, b, c, d \in \mathbb{Z}$, where the first signs on each side agree, and the second signs agree. This is the most general phase function on $\mathbb{Z} \times \mathbb{Z}$.

(7) For $z \in \mathbb{C}, \Gamma_{z}=\mathbb{R} \times \mathbb{R}$ and $\omega_{z}((a, b),(c, d))=\exp (i z(a d-b c))$ for $a, b, c, d \in$ $\mathbb{R}$. This is the most general continuous complex phase function on $\mathbb{R} \times \mathbb{R}$.

(8) For $z, w \in \mathbb{C}, \Gamma_{z, w}=\mathbb{C} \times \mathbb{C}$ and $\omega_{z, w}((a, b),(c, d))=\exp (z \Re(a d-b c)$ $+w \Im(a d-b c))$ for $a, b, c, d \in \mathbb{C}$. The phase function $\omega_{z, w}=\omega_{z, i z}$ is the most general complex analytic phase function on $\mathbb{C} \times \mathbb{C}$, and $\omega_{z, w}=\omega_{z,-i z}$ is the most general complex antianalytic phase function on $\mathbb{C} \times \mathbb{C}$.

Some general properties of phase functions, consequent to (2.1), are as follows:

- $\omega(\alpha, \beta)$ is nonzero for all $\alpha, \beta \in \Gamma$,

- $\omega(\alpha+\beta, \gamma)=\omega(\alpha, \gamma) \omega(\beta, \gamma)$,

- $\omega(\alpha, \alpha)= \pm 1$ for all $\alpha \in \Gamma$,

- $\omega(\alpha+\beta, \alpha+\beta)=\omega(\alpha, \alpha) \omega(\beta, \beta)$,

- $\omega(0, \alpha)=\omega(\alpha, 0)=1$,

- for given $\alpha$, the maps $\beta \mapsto \omega(\alpha, \beta)$ and $\beta \mapsto \omega(\beta, \alpha)$ are one-dimensional representations of $\Gamma$ in $\mathbb{F}$. 
Let an element $\alpha \in \Gamma$ be called even (respectively odd) if $\omega(\alpha, \alpha)=1$ (respectively -1 ). The sum of 2 even elements or 2 odd elements of $\Gamma$ is even. The sum of an even element and an odd element of $\Gamma$ is odd. Note that either all elements of $\Gamma$ are even, or the distinction partitions $\Gamma$ into two cosets (the set of odd elements form a coset of the group of even elements).

The phase functions on a group $\Gamma$ form an Abelian group: if $\omega$ and $\widetilde{\omega}$ are phase functions, then their product is defined by

$$
(\omega \widetilde{\omega})(\alpha, \beta)=\omega(\alpha, \beta) \widetilde{\omega}(\alpha, \beta) .
$$

The identity of this group is the trivial phase function:

$$
\omega_{e}(\alpha, \beta) \equiv 1,
$$

and the multiplicative inverse is given by

$$
\left(\omega^{-1}\right)(\alpha, \beta)=(\omega(\alpha, \beta))^{-1} .
$$

Given $(\Gamma, \omega)$, define $\psi: \Gamma \rightarrow \mathbb{Z}_{2}$ by

$$
\psi(\alpha)= \begin{cases}0, & \omega(\alpha, \alpha)=1, \\ 1, & \omega(\alpha, \alpha)=-1,\end{cases}
$$

then $\psi$ is a group morphism by the Property 3 above, and $\psi(\alpha)=0$ if $\alpha$ is even, $\psi(\alpha)=1$ if $\alpha$ is odd. Define $\omega_{0}: \Gamma \times \Gamma \rightarrow \mathbb{F}^{\times}$(where $\mathbb{F}^{\times}$is the multiplicative group of nonzero elements in $\mathbb{F}$ ) by

$$
\omega_{0}(\alpha, \beta)=(-1)^{\psi(\alpha) \psi(\beta)},
$$

then $\omega_{0}$ is a phase function and

$$
\omega_{0}(\alpha, \alpha)=\omega(\alpha, \alpha), \alpha \in \Gamma .
$$

Also,

$$
\omega_{0}(\alpha, \beta)=\left\{\begin{array}{l}
1, \quad \alpha \text { even, or } \beta \text { even } \\
-1, \quad \alpha, \beta \text { both odd. }
\end{array}\right.
$$

For $(\Gamma, \omega)$, let $\eta=\omega_{0} \omega^{-1}$, then $\eta$ is a phase function, $\eta(\alpha, \alpha)=1$ for all $\alpha \in \Gamma$, and

$$
\eta(\alpha, \beta)= \begin{cases}\frac{1}{\omega(\alpha, \beta)}, & \alpha \text { even, or } \beta \text { even, } \\ \frac{-1}{\omega(\alpha, \beta)}, & \alpha, \beta \text { both odd. }\end{cases}
$$


Examples. (1) For $(\Gamma, \omega)=\left(\Gamma_{S}, \omega_{S}\right)$, then $\omega_{0}=\omega_{S}$, and $\eta$ is trivial, that is, $\eta=\omega_{e}$.

(2) For $(\Gamma, \omega)=\left(\Gamma_{Q}, \omega_{Q}\right)$, then $\omega_{0}$ is trivial, that is, $\omega_{0}=\omega_{e}$, and $\eta=\omega_{Q}$.

(3) For $(\Gamma, \omega)=\left(\Gamma_{S Q}, \omega_{S Q}\right)$, then

$$
\begin{aligned}
\omega_{0}(\alpha, \beta) & =(-1)^{\alpha_{1} \beta_{1}}, \\
\eta(\alpha, \beta) & =(-1)^{\alpha_{2} \beta_{3}+\alpha_{3} \beta_{2}} .
\end{aligned}
$$

(4) For $(\Gamma, \omega)=\left(\Gamma_{C, n}, \omega_{C, n}\right)$, then $\omega_{0}$ is trivial, that is, $\omega_{0}=\omega_{\mathrm{e}}$, and $\eta=\omega_{C, n}$.

(5) For $(\Gamma, \omega)=\left(\Gamma_{n}, \omega_{n}\right)$, then $\omega_{0}$ is trivial, that is, $\omega_{0}=\omega_{e}$, and $\eta(\alpha, \beta)=$ $\zeta_{n}^{\alpha_{2} \beta_{1}-\alpha_{1} \beta_{2}}$.

(6) For $(\Gamma, \omega)=\left(\Gamma_{t}^{++}, \omega_{t}^{++}\right)$, then $\omega_{0}$ is trivial, that is, $\omega_{0}=\omega_{e}$, and $\eta((a, b),(c, d))$ $=t^{b c-a d}$. For $(\Gamma, \omega)=\left(\Gamma_{t}^{+-}, \omega_{t}^{+-}\right)$, then $\omega_{0}((a, b),(c, d))=(-1)^{b d}$, and $\eta((a, b),(c, d))$ $=t^{b c-a d}$. For $(\Gamma, \omega)=\left(\Gamma_{t}^{-+}, \omega_{t}^{-+}\right)$, then $\omega_{0}((a, b),(c, d))=(-1)^{a c}$, and $\eta((a, b),(c, d))$ $=t^{b c-a d}$. For $(\Gamma, \omega)=\left(\Gamma_{t}^{--}, \omega_{t}^{--}\right)$, then $\omega_{0}((a, b),(c, d))=(-1)^{(a+b)(c+d)}$, and $\eta((a, b),(c, d))=(-t)^{b c-a d}$.

(7) For $(\Gamma, \omega)=\left(\Gamma_{z}, \omega_{z}\right)$, then $\omega_{0}$ is trivial, that is, $\omega_{0}=\omega_{e}$, and $\eta((a, b),(c, d))=$ $\exp (i z(b c-a d))$.

(8) For $(\Gamma, \omega)=\left(\Gamma_{z, w}, \omega_{z, w}\right)$, then $\omega_{0}$ is trivial, that is, $\omega_{0}=\omega_{e}$, and $\eta((a, b),(c, d))$ $=\exp (z \Re(b c-a d)+w \Im(b c-a d))$.

(9) As a final example, if $(\Gamma, \omega)=\left(\Gamma_{S}, \omega_{S}\right)^{\times n}$, so that $\Gamma=\mathbb{Z}_{2}^{\times n}\left(=\Gamma_{C, n}\right)$ and $\omega(\alpha, \beta)=(-1)^{\sum_{i} \alpha_{i} \beta_{i}}$, then

$$
\begin{aligned}
\omega_{0}(\alpha, \beta) & =(-1)^{\left(\sum_{i} \alpha_{i}\right)\left(\sum_{j} \beta_{j}\right)} \\
\eta(\alpha, \beta) & =\omega_{C, n}(\alpha, \beta) .
\end{aligned}
$$

\section{Colour Structures}

We now review the definitions and properties of important colour structures, specifically, colour algebras, colour coalgebras, colour bialgebras, Hopf colour algebras, and Lie colour algebras.

(I) Colour Algebras.

A graded (or colour) space $M$ over the field $\mathbb{F}$ is a vector space over $\mathbb{F}$ such that

$$
M=\bigoplus_{\mu \in \Gamma} M_{\mu} .
$$

If $M$ and $N$ are colour spaces over $\mathbb{F}$, then there is a natural graded twist $T: M \otimes N \rightarrow$ $N \otimes M$ defined by

$$
T(m \otimes n)=\omega(\mu, \nu) n \otimes m, m \in M_{\mu}, n \in N_{\nu}
$$


which is distinct from the nongraded twist $\bar{T}$ given by

$$
\bar{T}(m \otimes n)=n \otimes m .
$$

A colour algebra is a graded unital associative algebra (an algebra with consistent grading), so that

$$
\mathbb{F} \subseteq A_{0}, \quad A_{\alpha} A_{\beta} \subseteq A_{\alpha+\beta}
$$

The colour tensor product of two colour algebras has product determined by

$$
A \otimes B \otimes A \otimes B \stackrel{1 \otimes T \otimes 1}{\longrightarrow} A \otimes A \otimes B \otimes B \stackrel{M \otimes M}{\longrightarrow} A \otimes B,
$$

so that if $x \in A, z \in B_{\zeta}, x^{\prime} \in A_{\xi^{\prime}}, z^{\prime} \in B$, then

$$
(x \otimes z)\left(x^{\prime} \otimes z^{\prime}\right)=\omega\left(\zeta, \xi^{\prime}\right) x x^{\prime} \otimes z z^{\prime} .
$$

This contrasts with the nongraded tensor product $A \underline{\otimes} B$ in which the product is given by

$$
(x \underline{\otimes} z)\left(x^{\prime} \underline{\otimes} z^{\prime}\right)=x x^{\prime} \underline{\otimes} z z^{\prime} .
$$

The colour tensor product and the nongraded tensor product are generally not isomorphic as algebras.

If $A$ is a colour unital algebra then an $A$-module $M$ is called a graded $A$-module if $M$ is a graded space over $\mathbb{F}$ (graded with respect to the same grading group $\Gamma$ as $A)$, and

$$
A_{\alpha} M_{\mu} \subseteq M_{\alpha+\mu}, \quad \alpha, \mu \in \Gamma .
$$

If $A, B$ are unital colour algebras, $M$ is a graded $A$-module, and $N$ is a graded $B$-module, then $M \otimes N$ is a graded $A \otimes B$-module, and the action of $A \otimes B$ on $M \otimes N$ is determined by

$$
A \otimes B \otimes M \otimes N \stackrel{1 \otimes T \otimes 1}{\longrightarrow} A \otimes M \otimes B \otimes N \longrightarrow M \otimes N,
$$

where $A \otimes M \rightarrow M$ and $B \otimes N \rightarrow N$ are given by the actions of $A$ on $M$, and $B$ on $N$, respectively. In particular, if $x \in A, z \in B_{\zeta}, m \in M_{\mu}, n \in N$, then

$$
(x \otimes z)(m \otimes n)=\omega(\zeta, \mu) x m \otimes z n .
$$




\section{(iI) Colour Coalgebras.}

A colour coalgebra is a graded counital coassociative coalgebra (a coalgebra with consistent grading), so that, if $\varepsilon$ and $\Delta$ denote the counit and coproduct respectively, then

$$
\begin{gathered}
\varepsilon\left(A_{\alpha}\right)=0, \alpha \neq 0, \\
\Delta\left(A_{\alpha}\right) \subseteq(A \otimes A)_{\alpha}=\bigoplus_{\beta+\gamma=\alpha} A_{\beta} \otimes A_{\gamma} .
\end{gathered}
$$

The colour tensor product of two colour coalgebras has coproduct determined by $A \otimes B \stackrel{\Delta \otimes \Delta}{\longrightarrow} A \otimes A \otimes B \otimes B \stackrel{1 \otimes T \otimes 1}{\longrightarrow} A \otimes B \otimes A \otimes B$,

so that

$$
\Delta(x \otimes z)=\sum_{(x),(z)} \omega\left(\xi_{(2)}, \zeta_{(1)}\right)\left(x_{(1)} \otimes z_{(1)}\right) \otimes\left(x_{(2)} \otimes z_{(2)}\right)
$$

where in a specific term in the sum, $x_{(2)} \in A_{\xi_{(2)}}, z_{(1)} \in B_{\zeta_{(1)}}$, some $\xi_{(2)}, \zeta_{(1)} \in$ $\Gamma$ (Sweedler's notation [16] is being used here). Note that this contrasts with the nongraded tensor product $A \underline{\otimes} B$ in which the coproduct is given by

$$
\underline{\Delta}(x \underline{\otimes} z)=\sum_{(x),(z)}\left(x_{(1)} \underline{\otimes} z_{(1)}\right) \underline{\otimes}\left(x_{(2)} \underline{\otimes} z_{(2)}\right),
$$

and that the colour tensor product and the nongraded tensor product are generally not isomorphic as coalgebras.

(III) Colour Bialgebras.

A colour bialgebra is a colour algebra and colour coalgebra with consistent gradings in which $\Delta: A \rightarrow A \otimes A, \varepsilon: A \rightarrow \mathbb{F}$ are colour algebra morphisms and $M: A \otimes A \rightarrow A$, $u: \mathbb{F} \rightarrow A$ are colour coalgebra morphisms, where $M$ denotes the product on $A$, and $u$ denotes the unit embedding $\mathbb{F}$ into $A$ (so that $u(1)$ is the multiplicative identity). Note that since the colour tensor product $A \otimes A$ and the nongraded tensor product $A \otimes A$ are generally not isomorphic either as algebras or coalgebras, then a colour bialgebra generally does not have the structure of a bialgebra.

(iv) Hopf Colour Algebras.

A Hopf colour algebra $A$ is a colour bialgebra with a linear map (antipode) $S_{A}$ : $A \rightarrow A$ of grading zero such that

$$
m_{A}\left(S_{A} \otimes 1\right) \Delta_{A}=m_{A}\left(1 \otimes S_{A}\right) \Delta_{A}=u_{A} \varepsilon_{A} .
$$


The antipode $S_{A}$ reverses both the product and the coproduct, in other words,

$$
\begin{aligned}
& S_{A} m_{A}=m_{A} T\left(S_{A} \otimes S_{A}\right)=m_{A}^{\prime}\left(S_{A} \otimes S_{A}\right), \\
& \Delta_{A} S_{A}=\left(S_{A} \otimes S_{A}\right) T \Delta_{A}=\left(S_{A} \otimes S_{A}\right) \Delta_{A}^{\prime},
\end{aligned}
$$

or, in particular,

$$
S_{A}\left(x x^{\prime}\right)=\omega\left(\xi, \xi^{\prime}\right) S_{A}\left(x^{\prime}\right) S_{A}(x),
$$

for $x \in A_{\xi}$ and $x^{\prime} \in A_{\xi^{\prime}}$, and

$$
\Delta_{A}\left(S_{A}(x)\right)=\sum_{(x)} \omega\left(\xi_{(1)}, \xi_{(2)}\right) S_{A}\left(x_{(2)}\right) \otimes S_{A}\left(x_{(1)}\right),
$$

where $\xi_{(1)}$ and $\xi_{(2)}$ are as before.

(v) Lie Colour Algebras.

A Lie colour algebra $\mathcal{L}$ over $\mathbb{F}$ is a graded vector space endowed with a colour commutator of grading zero satisfying colour anticommutativity and the colour Jacobi identity, so that

$$
\begin{gathered}
\mathcal{L}=\bigoplus_{\alpha \in \Gamma} \mathcal{L}_{\alpha},\left[\mathcal{L}_{\alpha}, \mathcal{L}_{\beta}\right] \subseteq \mathcal{L}_{\alpha+\beta}, \\
{[a, b]=-\omega(\alpha, \beta)[b, a], a \in \mathcal{L}_{\alpha}, b \in \mathcal{L}_{\beta},} \\
\omega(\gamma, \alpha)[a,[b, c]]+\omega(\alpha, \beta)[b,[c, a]]+\omega(\beta, \gamma)[c,[a, b]]=0, \\
a \in \mathcal{L}_{\alpha}, b \in \mathcal{L}_{\beta}, c \in \mathcal{L}_{\gamma} .
\end{gathered}
$$

A homomorphism from a Lie colour algebra $\mathcal{L}$ to a Lie colour algebra $\mathcal{M}$, with the same grading group and phase function, is a linear map $f$ of degree zero (so that $\left.f\left(\mathcal{L}_{\alpha}\right) \subseteq \mathcal{M}_{\alpha}\right)$ such that

$$
f([a, b])=[f(a), f(b)], a, b \in \mathcal{L} .
$$

The universal enveloping algebra $U(\mathcal{L})$ of a Lie colour algebra is the unital associative algebra generated by $\mathcal{L}$ subject to

$$
a b-\omega(\alpha, \beta) b a=[a, b], \alpha \in \mathcal{L}_{\alpha}, b \in \mathcal{L}_{\beta} .
$$

The universal enveloping algebra $U(\mathcal{L})$ is a Hopf colour algebra with coproduct, counit and antipode determined by

$$
\begin{aligned}
\Delta(a) & =a \otimes 1+1 \otimes a, a \in \mathcal{L}, \\
\varepsilon(a) & =0, a \in \mathcal{L}, \\
S(a) & =-a, a \in \mathcal{L},
\end{aligned}
$$

so that if $V$ and $W$ are graded $\mathcal{L}$-modules, then $V \otimes W$ is an $\mathcal{L}$-module, with the action of $\mathcal{L}$ given by

$$
a(m \otimes n)=a m \otimes n+\omega(\alpha, \mu) m \otimes a n, a \in \mathcal{L}_{\alpha}, m \in V_{\mu}, n \in W .
$$

Also, the adjoint action of $a \in \mathcal{L}$ is determined by $(\operatorname{ad} a) \cdot x=[a, x]$. 


\section{KNown Results for Lie Colour Algebras}

Here, we review relevant work of Kleeman $[7,8,9]$, specifically his results establishing a general correspondence between Lie colour algebras and Lie superalgebras, along with embedding of a Lie superalgebra in the extended universal enveloping algebra of the Lie colour algebra (after the introduction of generators external to the Lie colour algebra).

For this Section, the notation for the colour commutator will be $\langle a, b\rangle$ in line with Kleeman's notation, so that in the universal enveloping algebra,

$$
\langle a, b\rangle=a b-\omega(\alpha, \beta) b a .
$$

If $\sigma: \Gamma \times \Gamma \rightarrow \mathbb{F}^{\times}$is a multiplier [11] on $\Gamma$, so that $\sigma(\alpha, \beta)$ is a nonzero scalar and

$$
\sigma(\alpha+\beta, \gamma) \sigma(\alpha, \beta)=\sigma(\alpha, \beta+\gamma) \sigma(\beta, \gamma), \quad \alpha, \beta, \gamma \in \Gamma,
$$

then define

$$
\omega^{\prime}(\alpha, \beta)=\sigma(\alpha, \beta) \sigma^{-1}(\beta, \alpha) \omega(\alpha, \beta) .
$$

Then $\omega^{\prime}$ is a phase function on $\Gamma$, and $\mathcal{L}$ can be regarded as a Lie colour algebra $\mathcal{L}^{\sigma}$ with grading group and phase function $\left(\Gamma, \omega^{\prime}\right)$ with new colour commutator $\langle a, b\rangle_{\sigma}$ defined by

$$
\langle a, b\rangle_{\sigma}=\sigma(\alpha, \beta)\langle a, b\rangle, a \in \mathcal{L}_{\alpha}, b \in \mathcal{L}_{\beta}
$$

If $\sigma$ satisfies the stronger conditions

$$
\begin{aligned}
& \sigma(\alpha+\beta, \gamma)=\sigma(\alpha, \gamma) \sigma(\beta, \gamma), \\
& \sigma(\alpha, \beta+\gamma)=\sigma(\alpha, \beta) \sigma(\alpha, \gamma),
\end{aligned}
$$

then let $U^{\sigma}(\mathcal{L})$ be the unital associative algebra generated by the universal enveloping algebra $U(\mathcal{L})$ and further generators $K^{\sigma}(\alpha)(\alpha \in \Gamma)$ of grading zero $\left(K^{\sigma}(\alpha)\right.$ are called Klein operators by Kleeman [9]), subject to

$$
\begin{aligned}
& K^{\sigma}(\alpha) K^{\sigma}(\beta)=K^{\sigma}(\alpha+\beta), K^{\sigma}(0)=1, \\
& K^{\sigma}(\alpha) b=\sigma(\beta, \alpha) b K^{\sigma}(\alpha), b \in(U(\mathcal{L}))_{\beta} .
\end{aligned}
$$

A graded $\mathcal{L}$-module $M$ can be endowed with the structure of an $U^{\sigma}(\mathcal{L})$-module by defining

$$
K^{o}(\alpha) m=\sigma(\mu, \alpha) m, m \in M_{\mu}
$$


For $a \in \mathcal{L}$, define

$$
a^{\sigma}=K^{\sigma}(-\alpha) a, \quad a \in \mathcal{L}_{\alpha},
$$

and extend by linearity, then

$$
\left\langle a^{\sigma}, b^{\sigma}\right\rangle_{\sigma}=\sigma(\alpha, \beta)\langle a, b\rangle^{\sigma},
$$

so that $\mathcal{L}^{\sigma}$ can be embedded into $U^{\sigma}(\mathcal{L})$, and $U^{\sigma}\left(\mathcal{L}^{\sigma}\right)$ is isomorphic to $U^{\sigma}(\mathcal{L})$. In other words, the Lie colour algebra $\mathcal{L}^{\sigma}$ is embedded in the algebra $U^{\sigma}(\mathcal{L})$ generated by the universal enveloping algebra $U(\mathcal{L})$ and the Klein operators $K^{\sigma}(\alpha)$, and the Lie colour algebra $\mathcal{L}$ is embedded in the algebra $U^{\sigma}\left(\mathcal{L}^{\sigma}\right)$ generated by the universal enveloping algebra $U\left(\mathcal{L}^{\sigma}\right)$ and the Klein operators $K^{\sigma}(\alpha)$ : they can be embedded in the extended universal enveloping algebra of each other.

A finitely generated Abelian group $\Gamma$ possesses a unique (up to isomorphism) decomposition given by

$$
\begin{aligned}
\Gamma & =\Gamma_{p_{1}} \times \Gamma_{p_{2}} \times \ldots \Gamma_{p_{n}} \times \mathbb{Z} \times \cdots \times \mathbb{Z} \\
& \cong \Gamma_{p_{1}} \oplus \Gamma_{p_{2}} \oplus \ldots \Gamma_{p_{n}} \oplus \mathbb{Z} \oplus \cdots \oplus \mathbb{Z}
\end{aligned}
$$

where $\Gamma_{p_{i}}$ is an Abelian $p_{i}$-subgroup of $\Gamma$ (where $p_{i}$ are prime). Each $\Gamma_{p_{i}}$ can be further decomposed into cyclic subgroups:

$$
\begin{aligned}
\Gamma_{p_{i}} & =\mathbb{Z}_{\left(p_{i}\right)^{r_{1}}} \times \cdots \times \mathbb{Z}_{\left(p_{i}\right)^{r_{m}}} \\
& \cong \mathbb{Z}_{\left(p_{i}\right)^{r_{1}}} \oplus \cdots \otimes \mathbb{Z}_{\left(p_{i}\right)^{r_{m}}} .
\end{aligned}
$$

In the case of a finite group, the copies of $\mathbb{Z}$ are omitted in (4.10).

Let $\left\{q_{i}\right\}$ be the set of cyclic generators of $\Gamma$, so that $q_{i}$ is the generator of either one of the copies of $\mathbb{Z}_{\left(p_{j}\right)^{r_{k}}}$ or of one of the copies of $\mathbb{Z}$. Define $\omega_{i j}$ (here, Kleeman's notation $E_{i j}$ is not used since there is the possibility of confusion with the generators of $g l\left(\mu_{1}, \ldots, \mu_{n}\right)$ below) by

$$
\omega_{i j}=\omega\left(q_{i}, q_{j}\right) \neq 0
$$

Then

$$
\omega(\alpha, \beta)=\prod_{i}\left(\omega_{i i}\right)^{n_{i} m_{i}} \prod_{j<k}\left(\omega_{j k}\right)^{n_{j} m_{k}-n_{k} m_{j}}
$$

where

$$
\begin{aligned}
& \alpha=\sum_{i} n_{i} q_{i}, \\
& \beta=\sum_{i} m_{i} q_{i} .
\end{aligned}
$$


If $q_{i}$ generates a copy of $\mathbb{Z}_{\left(p_{a}\right)^{r_{b}}}$ and $q_{j}$ generates a copy of $\mathbb{Z}_{\left(p_{c}\right)^{r_{d}}}(i \neq j)$, then (1) If $a=c$, then $\omega_{i j}$ is a $v_{i j}$-th root of unity, where

$$
v_{i j}=\min \left(\left(p_{a}\right)^{r_{b}},\left(p_{a}\right)^{r_{d}}\right)=\left(p_{a}\right)^{\min \left(r_{b}, r_{d}\right)}
$$

(2) If $a \neq c$, then $\omega_{i j}=1$.

If $q_{i}$ generates a copy of $\mathbb{Z}_{\left(p_{a}\right)^{r_{b}}}$ and $q_{j}$ generates a copy of $\mathbb{Z}$, then $\omega_{i j}$ is a $\left(p_{a}\right)^{r_{b}}$-th root of unity.

If $q_{i}$ generates a copy of $\mathbb{Z}$ and $q_{j}$ generates a copy of $\mathbb{Z}_{\left(p_{a}\right)^{r_{b}}}$, then $\omega_{i j}$ is a $\left(p_{a}\right)^{r_{b}}$-th root of unity.

If $q_{i}$ generates a copy of $\mathbb{Z}$ and $q_{j}$ generates a copy of $\mathbb{Z}(i \neq j)$, then $\omega_{i j}$ is an arbitrary nonzero element of $\mathbb{F}$.

If $q_{i}$ generates a copy of $\mathbb{Z}_{\left(p_{a}\right)^{r_{b}}}$, then

(1) If $p_{a}=2$, then $\omega_{i i}= \pm 1$;

(2) If $p_{a} \neq 2$, then $\omega_{i i}=1$.

If $q_{i}$ generates a copy of $\mathbb{Z}$, then $\omega_{i i}= \pm 1$.

THEOREM. If $\Gamma$ is finitely-generated and $\omega$ is a phase function in $\Gamma$ such that $\omega(\alpha, \alpha)=1$ for all $\alpha \in \Gamma$, then there exists a function $\sigma: \Gamma \times \Gamma \rightarrow \mathbb{F}$ such that

$$
\begin{aligned}
\sigma(\alpha, \beta) \sigma^{-1}(\beta, \alpha) & =\omega(\alpha, \beta), \\
\sigma(\alpha+\beta, \gamma) & =\sigma(\alpha, \gamma) \sigma(\beta, \gamma), \\
\sigma(\alpha, \beta+\gamma) & =\sigma(\alpha, \beta) \sigma(\alpha, \gamma) .
\end{aligned}
$$

Proof: By (4.13),

$$
\omega(\alpha, \beta)=\prod_{i}\left(\omega_{i i}\right)^{n_{i} m_{i}} \prod_{j<k}\left(\omega_{j k}\right)^{n_{j} m_{k}-n_{k} m_{j}},
$$

for

$$
\begin{aligned}
& \alpha=\sum_{i} n_{i} q_{i} \\
& \beta=\sum_{i} m_{i} q_{i},
\end{aligned}
$$

where $\omega_{i j}=\omega\left(q_{i}, q_{j}\right)$. Since $\omega(\alpha, \alpha)=1$ for all $\alpha \in \Gamma$, then, taking $\alpha=q_{i}, \omega_{i i}=1$ for all $i$, so

$$
\omega(\alpha, \beta)=\prod_{j<k}\left(\omega_{j k}\right)^{n_{j} m_{k}-n_{k} m_{j}}
$$


The function

$$
\sigma(\alpha, \beta)=\prod_{j<k}\left(\omega_{j k}\right)^{n_{j} m_{k}}
$$

satisfies the conditions (4.16).

By defining $\omega_{0}$ and $\eta$ as in Section 2, then $\eta$ is a phase function such that $\eta(\alpha, \alpha)=$ 1 for all $\alpha \in \Gamma$, and so if $\Gamma$ is finitely-generated, there exists a function $\sigma: \Gamma \times \Gamma \rightarrow \mathbb{F}$ such that

$$
\begin{aligned}
\sigma(\alpha, \beta) \sigma^{-1}(\beta, \alpha) & =\eta(\alpha, \beta), \\
\sigma(\alpha+\beta, \gamma) & =\sigma(\alpha, \gamma) \sigma(\beta, \gamma), \\
\sigma(\alpha, \beta+\gamma) & =\sigma(\alpha, \beta) \sigma(\alpha, \gamma),
\end{aligned}
$$

so that $\omega_{0}(\alpha, \beta)=\sigma(\alpha, \beta) \sigma^{-1}(\beta, \alpha) \omega(\alpha, \beta)$.

If $\omega$ is a phase function and $\sigma: \Gamma \times \Gamma \rightarrow \mathbb{F}$ satisfies

$$
\begin{aligned}
\sigma(\alpha, \beta) \sigma^{-1}(\beta, \alpha) \omega(\alpha, \beta) & =\omega_{0}(\alpha, \beta), \\
\sigma(\alpha+\beta, \gamma) & =\sigma(\alpha, \gamma) \sigma(\beta, \gamma), \\
\sigma(\alpha, \beta+\gamma) & =\sigma(\alpha, \beta) \sigma(\alpha, \gamma),
\end{aligned}
$$

then $\mathcal{L}^{\sigma}$ becomes a Lie superalgebra under $\langle\cdot, \cdot\rangle_{\sigma}$, and the Lie superalgebra can be embedded into $U^{\sigma}(\mathcal{L})$. It follows that if $\Gamma$ is a finitely-generated Abelian group, then for an appropriate choice of $\sigma$, a Lie superalgebra $\mathcal{L}^{\sigma}$ can be embedded into the extended universal enveloping algebra $U^{\sigma}(\mathcal{L})$.

On the other hand, suppose that $\Gamma$ is not finitely-generated. Since there is no guarantee that a function $\sigma$ satisfying (4.22) exists, then there may not exist a function $\sigma$ such that a Lie superalgebra can be embedded into $U^{\sigma}(\mathcal{L})$. Suppose $\mathcal{L}$ is a finitelygenerated colour Lie algebra (with homogeneous generators). Let $\left\{x_{i}: i=1, \ldots, n\right\}$ be such a set of generators, and suppose $x_{i} \in \mathcal{L}_{\xi_{i}}$ for $i=1, \ldots, n$. By defining $\Gamma_{\mathcal{L}}=\sum_{i=1}^{n} \mathbb{Z} \xi_{i}$ and $\omega_{\mathcal{L}}$ to be the restriction of $\omega$ on $\Gamma_{\mathcal{L}} \times \Gamma_{\mathcal{L}}$, then $\mathcal{L}$ is a Lie colour algebra with respect to $\left(\Gamma_{\mathcal{L}}, \omega_{\mathcal{L}}\right)$. Since $\Gamma_{\mathcal{L}}$ is finitely-generated, then a suitable function $\sigma$ can be defined on $\Gamma_{\mathcal{L}} \times \Gamma_{\mathcal{L}}$, so that, introducing $K^{\sigma}(\alpha)$ for $\alpha \in \Gamma_{\mathcal{L}}$ satisfying

$$
\begin{aligned}
K^{\sigma}(\alpha) K^{\sigma}(\beta) & =K^{\sigma}(\alpha+\beta), K^{\sigma}(0)=1, \\
K^{\sigma}(\alpha) b & =\sigma(\beta, \alpha) b K^{\sigma}(\alpha), b \in(U(\mathcal{L}))_{\beta}, \beta \in \Gamma_{\mathcal{L}}
\end{aligned}
$$

then there is a Lie superalgebra embedded in $U^{\sigma}(\mathcal{L})$ where $U^{\sigma}(\mathcal{L})$ is the unital associative algebra generated by $U(\mathcal{L})$ and $K^{\sigma}$. Thus it follows that such a Lie superalgebra is guaranteed to exist if $\mathcal{L}$ is finitely-generated by homogeneous elements. 
The essential feature of these embeddings of a Lie superalgebra into an algebra containing a given Lie colour algebra is that the introduction of new generators from outside the universal enveloping algebra was required. We shall show in Section 8 that a Lie superalgebra can be defined within the context of the universal enveloping algebra of colour $g l(n)$ purely in terms of the generators of $g l(n)$, so that there is no need to introduce anything external to the algebra to obtain the Lie superalgebra.

The following results, also related to those of Kleeman $[7,8,9]$, may be noted.

Define

$$
\Gamma_{0}=\{\alpha \in \Gamma: \omega(\alpha, \beta)=1 \forall \beta \in \Gamma\},
$$

and call $\Gamma$ reduced if $\Gamma_{0}=0$ [9]. In [10], the term "nondegenerate" is equivalent to "reduced" here. Set $\Gamma^{r}=\Gamma / \Gamma_{0}$, then $\Gamma^{r}$ inherits a phase function $\omega^{r}$ from $\Gamma$, so that

$$
\omega^{r}\left(\alpha+\Gamma_{0}, \beta+\Gamma_{0}\right)=\omega(\alpha, \beta) .
$$

Note that $\left(\Gamma^{r}\right)_{0}=0$.

The following theorem is inspired by a theorem of Kleeman on canonical groups and phase functions.

THEOREM. If $(\Gamma, \omega)$ is a finite reduced Abelian group and phase function, then $(\Gamma, \omega)$ can be decomposed as

$$
(\Gamma, \omega)=\left(\Gamma_{p_{1}}, \omega_{p_{1}}\right) \times \cdots \times\left(\Gamma_{p_{n}}, \omega_{p_{n}}\right),
$$

where each $p_{i}$ is prime and each $\left(\Gamma_{p_{i}}, \omega_{p_{i}}\right)$ can be decomposed as

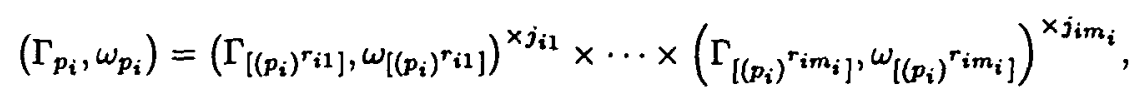

for $p_{i}$ odd, where $r_{i 1}, \ldots, r_{i m_{i}}$ are distinct positive integers, and $j_{i 1}, \ldots, j_{i m_{i}}$ are positive integers, where for $n \in \mathbb{N}$,

$$
\begin{aligned}
\Gamma_{[n]} & =\mathbb{Z}_{n} \times \mathbb{Z}_{n}, \\
\omega_{[n]}((a, b),(c, d)) & =\xi_{n}^{a d-b c}, a, b, c, d \in \mathbb{Z}_{n} .
\end{aligned}
$$

Here $\xi_{n}$ is a primitive $n$-th root of unity, so $\left(\Gamma_{[n]}, \omega_{[n]}\right)$ is meaningful only if $\mathbb{F}$ has primitive $n$-th roots of unity. Specifically, if $\mathbb{F}$ is a finite field $(G F(q)$, for $q$ a prime power), it is meaningful only if $q-1$ is divisible by $n$, and if $\mathbb{F}$ has characteristic $p$, then $\left(\Gamma_{[n]}, \omega_{[n]}\right)$ is meaningless if $n$ is divisible by $p$. In the case of $p_{i}=2$,

$$
\begin{aligned}
& \left(\Gamma_{2}, \omega_{2}\right)=\left(\Gamma_{\left[2^{\left.r_{i 1}\right]},\right.}, \varepsilon_{\left[r_{i 1}\right]}^{a_{1}} \omega_{\left[2^{r_{i 1}}\right]}\right) \times\left(\Gamma_{\left[2^{r_{i 1}}\right]}, \omega_{\left[2^{\left.r_{i 1}\right]}\right.}\right)^{\times j_{i 1}-1} \times \cdots \\
& \cdots \times\left(\Gamma_{\left[2^{r_{i m}}\right]}, \varepsilon_{\left[r_{i m_{i}}\right]}^{a m_{i}} \omega_{\left[2^{r_{i m_{1}}}\right]}\right) \times\left(\Gamma_{\left[2^{r_{i m_{i}}}\right]}, \omega_{\left[2^{r_{i m_{i}}}\right]}\right)^{\times j_{i m_{i}}-1} \times(\Gamma(2), \omega(2)),
\end{aligned}
$$


where $1, r_{i 1}, \ldots, r_{i m_{i}}$ are distinct positive integers, $j_{i 1}, \ldots, j_{i m_{i}}$ are positive integers, $a_{1}, \ldots, a_{m_{i}} \in \mathbb{Z}_{2}, \varepsilon_{[m]}$ is the phase function on $\mathbb{Z}_{2^{m}} \times \mathbb{Z}_{2^{m}}$ defined by

$$
\varepsilon_{[m]}((a, b),(c, d))=(-1)^{a c}, a, b, c, d \in \mathbb{Z}_{2^{m}},
$$

and either

$$
(\Gamma(2), \omega(2))=\left(\Gamma_{[2]}, \omega_{[2]}\right)^{\times m},
$$

where $m$ is a non-negative integer, or

$$
(\Gamma(2), \omega(2))=\left(\Gamma_{S}, \omega_{S}\right)^{\times m}
$$

where $m$ is a positive integer. In the case of (4.31), at most one of $a_{1}, \ldots, a_{m_{i}}$ is nonzero, and in the case of (4.32), all of $a_{1}, \ldots, a_{m_{i}}$ are zero.

\section{AN EXAMPLE: COLOUR $g l(n)$}

In this Section, we give a general definition for colour $g l(n)$, and introduce its vector representation and its contragredient representation.

Let $\mu_{1}, \ldots \mu_{n} \in \Gamma$ be given. The Lie colour algebra $g l\left(\mu_{1}, \ldots, \mu_{n}\right)$ has basis $\left\{E^{i}{ }_{j}: i, j=1, \ldots, n\right\}$, where $E^{i}{ }_{j} \in\left(g l\left(\mu_{1}, \ldots, \mu_{n}\right)\right)_{\mu_{i}-\mu_{j}}$, with colour commutator

$$
\left[E^{i}{ }_{j}, E^{k}{ }_{l}\right]=\delta_{j}^{k} E^{i}{ }_{l}-\delta_{l}^{i} \omega\left(\mu_{i}, \mu_{i}\right) \omega\left(\mu_{i}, \mu_{k}\right) \omega\left(\mu_{k}, \mu_{j}\right) \omega\left(\mu_{j}, \mu_{i}\right) E^{k}{ }_{j}
$$

The Lie colour algebra $g l\left(\mu_{1}, \ldots, \mu_{n}\right)$ is isomorphic to the Lie colour algebra of matrices over a graded $n$-dimensional vector space, $V$, with a basis $\left\{e^{k}\right\}$, where $e^{k} \in$ $V_{\mu_{k}}$ for $k=1, \ldots, n$. The action of $E^{i}{ }_{j}$ on this basis is determined by

$$
E^{i}{ }_{j} e^{k}=\delta_{j}^{k} e^{i},
$$

so that the matrix elements of $E^{i}{ }_{j}$ are given by

$$
\left(E^{i}\right)_{k}{ }^{l}=\delta_{k}^{i} \delta_{j}^{l} \text {. }
$$

Call the representation on $V$ the vector representation. The contragredient representation is defined on $V^{*}$, where the dual basis $\left\{e_{k}^{*}\right\}$ (for which $\left(e_{k}^{*}, e^{l}\right)=\delta_{k}^{l}$ ) satisfies $e_{k}^{*} \in V_{-\mu_{k}}^{*}$, with the action given by

$$
E^{i}{ }_{j} e_{k}^{*}=-\delta_{k}^{i} \omega\left(\mu_{i}, \mu_{i}\right) \omega\left(\mu_{j}, \mu_{i}\right) e_{j}^{*}
$$

The Lie colour algebras $g l\left(\mu_{1}, \ldots, \mu_{n}\right)$ and $g l\left(\mu_{1}+\nu, \ldots, \mu_{n}+\nu\right)$ have a natural isomorphism for all $\nu \in \Gamma\left(E^{i}{ }_{j} \leftrightarrow E^{i}{ }_{j}\right)$, and for $\sigma \in \mathcal{S}_{n}$, there is an isomorphism of $g l\left(\mu_{1}, \ldots, \mu_{n}\right)$ and $g l\left(\mu_{\sigma(1)}, \ldots, \mu_{\sigma(n)}\right)$ determined by $E^{i}{ }_{j} \leftrightarrow{E^{\sigma^{-1}(i)} \sigma^{-1}(j)}$.

In the case where $\mu_{1}$ and $\mu_{2}$ have the same parity (so that $\mu_{1}-\mu_{2}$ is even), the generators of $g l\left(\mu_{1}, \mu_{2}\right)$ satisfy the same relations as the corresponding generators for $g l(2)$. Similarly, in the case where $\mu_{1}$ and $\mu_{2}$ have opposite parity (so that $\mu_{1}-\mu_{2}$ is odd), the generators of $g l\left(\mu_{1}, \mu_{2}\right)$ satisfy the same relations as the corresponding generators for $g l(1 \mid 1)$. 


\section{Colour Bosons and Fermions}

Here, we introduce a realisation of colour $g l(n)$ in terms of colour boson and fermion generators.

Just as there is a realisation of $g l(n)$ in terms of ordinary boson operators, and of $g l(m \mid n)$ in terms of ordinary boson and fermion operators, so there is a realisation of colour $g l(n)$ in terms of colour boson and fermion operators. Take the unital associative colour algebra generated by $\left\{a^{i}: i=1, \ldots, n\right\}$ (the creation operators) and $\left\{a_{i}: i=\right.$ $1, \ldots, n\}$ (the annihilation operators), where $a^{i}$ has grading $\mu_{i}$ and $a_{i}$ has grading $-\mu_{i}$, subject to

$$
\begin{aligned}
& {\left[a^{i}, a^{j}\right] \equiv a^{i} a^{j}-\omega\left(\mu_{i}, \mu_{j}\right) a^{j} a^{i}=0} \\
& {\left[a_{i}, a_{j}\right] \equiv a_{i} a_{j}-\omega\left(\mu_{i}, \mu_{j}\right) a_{j} a_{i}=0} \\
& {\left[a_{i}, a^{j}\right] \equiv a_{i} a^{j}-\omega\left(\mu_{j}, \mu_{i}\right) a^{j} a_{i}=\delta_{i}^{j}}
\end{aligned}
$$

Note that if $\mu_{i}$ is even, then

$$
a_{i} a^{i}-a^{i} a_{i}=1
$$

so that $a_{i}$ and $a^{i}$ are ordinary boson operators, and if $\mu_{i}$ is odd, then

$$
\begin{aligned}
\left(a^{i}\right)^{2} & =0, \\
\left(a_{i}\right)^{2} & =0, \\
a_{i} a^{i}+a^{i} a_{i} & =1,
\end{aligned}
$$

so that $a_{i}$ and $a^{i}$ are ordinary fermion operators. The generators of $g l\left(\mu_{1}, \ldots, \mu_{n}\right)$ can be realised in terms of colour boson and fermion operators by

$$
E^{i}{ }_{j}=a^{i} a_{j}
$$

The colour commutation relations between the realisations of $E^{i}{ }_{j}$ and the creation operators $\left\{a^{k}\right\}$ (the annihilation operators $\left\{a_{k}\right\}$ ) are then given by

$$
\begin{aligned}
& {\left[E^{i}{ }_{j}, a^{k}\right] \equiv E^{i}{ }_{j} a^{k}-\omega\left(\mu_{i}, \mu_{k}\right) \omega\left(\mu_{k}, \mu_{j}\right) a^{k} E^{i}{ }_{j}=\delta_{j}^{k} a^{i}} \\
& {\left[E^{i}{ }_{j}, a_{k}\right] \equiv E^{i}{ }_{j} a_{k}-\omega\left(\mu_{k}, \mu_{i}\right) \omega\left(\mu_{j}, \mu_{k}\right) a_{k} E^{i}{ }_{j}=-\delta_{k}^{i} \omega\left(\mu_{i}, \mu_{i}\right) \omega\left(\mu_{j}, \mu_{i}\right) a_{j},}
\end{aligned}
$$

so that $\left\{a^{k}\right\}$ form a vector operator, and $\left\{a_{k}\right\}$ form a contragredient operator.

Note that the colour bosons and fermions differ from the modular statistics operators of Green [3], which are also associated with Lie colour algebras. 


\section{UNCOLOURING OF BOSONS AND FERMIONS}

Here, we uncolour the colour boson and fermion operators in the realisation, using only operators within the "complete" universal enveloping algebra (so that the uncoloured bosons and fermions are defined completely in terms of the generators of the Lie colour algebra).

Take the colour Fock space for the colour boson and fermion operators. Define

$$
U[k]=\prod_{m=1}^{k-1}\left[\omega\left(\mu_{m}, \mu_{k}\right)^{E^{m}} \omega_{0}\left(\mu_{m}, \mu_{k}\right)^{E_{m}^{m}}\right],
$$

where $\omega_{0}$ is defined as in Section 2 (so $U[1]=1$ ), then

$$
U[k]^{-1} a^{l} U[k]= \begin{cases}\omega\left(\mu_{k}, \mu_{l}\right) \omega_{0}\left(\mu_{k}, \mu_{l}\right) a^{l}, & l<k, \\ a^{l}, & l \geqslant k,\end{cases}
$$

and

$$
U[k]^{-1} a_{l} U[k]= \begin{cases}\omega\left(\mu_{l}, \mu_{k}\right) \omega_{0}\left(\mu_{k}, \mu_{l}\right) a_{l}, & l<k \\ a_{l}, & l \geqslant k\end{cases}
$$

and

$$
U[k] U[l]=U[l] U[k]
$$

Define

$$
\begin{aligned}
& b^{i}=U[i] a^{i}, \\
& b_{i}=a_{i} U[i]^{-1},
\end{aligned}
$$

then

$$
\begin{aligned}
& b^{i} b^{j}-\omega_{0}\left(\mu_{i}, \mu_{j}\right) b^{j} b^{i}=0 \\
& b_{i} b_{j}-\omega_{0}\left(\mu_{i}, \mu_{j}\right) b_{j} b_{i}=0 \\
& b_{i} b^{j}-\omega_{0}\left(\mu_{i}, \mu_{j}\right) b^{j} b_{i}=\delta_{i}^{j}
\end{aligned}
$$

in particular, if $\mu_{i}$ is even or $\mu_{j}$ is even, then

$$
\begin{aligned}
b^{i} b^{j} & =b^{j} b^{i}, \\
b_{i} b_{j} & =b_{j} b_{i}, \\
b_{i} b^{j}-b^{j} b_{i} & =\delta_{i}^{j},
\end{aligned}
$$


and if $\mu_{i}$ and $\mu_{j}$ are both odd, then

$$
\begin{aligned}
b^{i} b^{j} & =-b^{j} b^{i}, \\
b_{i} b_{j} & =-b_{j} b_{i}, \\
b_{i} b^{j}+b^{j} b_{i} & =\delta_{i}^{j},
\end{aligned}
$$

so that $\left\{b^{i}\right\}$ and $\left\{b_{i}\right\}$ are standard boson and fermion operators. Let $F^{i}{ }_{j}=b^{i} b_{j}$, then

$$
\begin{aligned}
F^{i}{ }_{j} & =U[i] E^{i}{ }_{j} U[j]^{-1}, \\
F^{i}{ }_{i} & =E^{i}{ }_{i},
\end{aligned}
$$

so the Cartan elements remain unchanged, and

$$
\begin{aligned}
F^{i}{ }_{j} F^{k}{ }_{l}-\omega_{0}\left(\mu_{i}, \mu_{k}\right) & \omega_{0}\left(\mu_{l}, \mu_{i}\right) \omega_{0}\left(\mu_{k}, \mu_{j}\right) \omega_{0}\left(\mu_{j}, \mu_{l}\right) F^{k}{ }_{l} F^{i}{ }_{j} \\
& =\delta_{j}^{k} F^{i}{ }_{l}-\delta_{l} \omega_{0}\left(\mu_{i}, \mu_{i}\right) \omega_{0}\left(\mu_{i}, \mu_{k}\right) \omega_{0}\left(\mu_{k}, \mu_{j}\right) \omega_{0}\left(\mu_{j}, \mu_{i}\right) F^{k}{ }_{j}
\end{aligned}
$$

in particular, if $\mu_{i}$ and $\mu_{j}$ have the same parity, or if $\mu_{k}$ and $\mu_{l}$ have the same parity, then

$$
F^{i}{ }_{j} F_{l}-F^{k}{ }_{l} F_{j}^{i}=\delta_{j}^{k} F_{l}^{i}-\delta_{l}^{i} F^{k}{ }_{j}
$$

and if $\mu_{i}$ and $\mu_{j}$ have opposite parity and $\mu_{k}$ and $\mu_{l}$ have opposite parity, then

$$
F^{i}{ }_{j} F_{l}{ }_{l}+F^{k}{ }_{l} F_{j}{ }_{j}=\delta_{j}^{k} F^{i}{ }_{l}+\delta_{l}^{i} F^{k}{ }_{j},
$$

so that $\left\{F^{i}{ }_{j}\right\}$ span a Lie superalgebra isomorphic to $g l(p \mid q)$, where $p$ is the number of even $\mu_{i}$, and $q$ is the number of odd $\mu_{i}(p+q=n)$.

\section{UnCOLOURING OF Colour $g l(n)$}

Here, the method developed in Section 7 is generalised to arbitrary modules of colour $g l(n)$ within certain very wide-ranging categories (any weight module is a direct sum of modules from within these categories).

In a cyclic module generated by a homogeneous (with respect to the grading group Г) nonzero vector $w$ with weight $\lambda=\left(\lambda_{1}, \ldots, \lambda_{n}\right)$, so that $E^{i}{ }_{i} w=\lambda_{i} w$ for all $i$, every weight $\kappa=\left(\kappa_{1}, \ldots, \kappa_{n}\right)$ satisfies $\kappa_{i}-\lambda_{i} \in \mathbb{Z}$ for all $i$, that is, $\kappa-\lambda$ is integral. Furthermore, for all elements $\lambda \in \mathcal{H}^{*}$, where $\mathcal{H}$ is the Cartan subalgebra (the span of $E^{i}{ }_{i}$ ), let $\mathcal{O}_{\lambda}$ be the category of graded modules with a homogeneous weight basis (a basis in which every vector $v$ is homogeneous with respect to the grading group $\Gamma$ and has a weight $\kappa$ ) in which every weight differs from $\lambda$ by an integral element of $\mathcal{H}^{*}$ (so 
that $\mathcal{O}_{\lambda}$ and $\mathcal{O}_{\kappa}$ are identical if $\lambda-\kappa$ is integral). Every module with a homogeneous weight basis is decomposable into a direct sum of modules from individual categories.

Take a nontrivial module $W$ in the category $\mathcal{O}_{\lambda}$, so every weight differs from $\lambda$ by an integral element of $\mathcal{H}^{*}$. Note that if $z$ is nonzero, then $z^{E^{i}{ }_{i}-\lambda_{i}}$ is well-defined on $W$. Since $E^{i}{ }_{i}$ has grading zero, then $z^{E^{i}{ }_{i}-\lambda_{i}}$ has grading zero. Let $\zeta_{i j}$ be nonzero elements of $\mathbb{F}$ for $i, j=1, \ldots, n$. Define

$$
W_{\lambda}[k]=\prod_{m=1}^{n} \zeta_{k m}^{E^{m}{ }^{m-\lambda_{m}}}
$$

then $W_{\lambda}[i]$ has grading zero, and

$$
W_{\lambda}[i]^{-1} E^{j}{ }_{k} W_{\lambda}[i]=\zeta_{i j}^{-1} \zeta_{i k} E^{j}{ }_{k}
$$

and

$$
W_{\lambda}[i] W_{\lambda}[j]=W_{\lambda}[j] W_{\lambda}[i]
$$

Define

$$
F^{i}{ }_{j}=W_{\lambda}[i] E^{i}{ }_{j} W_{\lambda}[j]^{-1},
$$

so that, in particular, $F_{i}^{i}=E^{i}{ }_{i}$, so the Cartan elements remain unchanged, then $F^{i}{ }_{j}$ has grading $\mu_{i}-\mu_{j}$, and

$$
\begin{gathered}
F^{i}{ }_{j} F^{k}{ }_{l}-\zeta_{i k} \zeta_{k i}^{-1} \omega\left(\mu_{i}, \mu_{k}\right) \zeta_{l i} \zeta_{i l}^{-1} \omega\left(\mu_{l}, \mu_{i}\right) \zeta_{k j} \zeta_{j k}^{-1} \omega\left(\mu_{k}, \mu_{j}\right) \zeta_{j l} \zeta_{l j}^{-1} \omega\left(\mu_{j}, \mu_{l}\right) F^{k}{ }_{l} F^{i}{ }_{j} \\
\quad=\delta_{j}^{k} F^{i}{ }_{l}-\delta_{l}^{i} \omega\left(\mu_{i}, \mu_{i}\right) \zeta_{i k} \zeta_{k i}^{-1} \omega\left(\mu_{i}, \mu_{k}\right) \zeta_{k j} \zeta_{j k}^{-1} \omega\left(\mu_{k}, \mu_{j}\right) \zeta_{j i} \zeta_{i j}^{-1} \omega\left(\mu_{j}, \mu_{i}\right) F^{k}{ }_{j}
\end{gathered}
$$

If there exists a phase function $\eta$ such that

$$
\eta\left(\mu_{i}, \mu_{j}\right)=\zeta_{i j} \zeta_{j i}^{-1}, \quad i, j=1, \ldots, n,
$$

so that $\eta\left(\mu_{i}, \mu_{i}\right)=1$ for all $i$, then

$$
\begin{aligned}
F^{i}{ }_{j} F^{k}{ }_{l}-(\eta \omega)\left(\mu_{i}, \mu_{k}\right)(\eta \omega)\left(\mu_{l}, \mu_{i}\right)(\eta \omega)\left(\mu_{k}, \mu_{j}\right)(\eta \omega)\left(\mu_{j}, \mu_{l}\right) F^{k}{ }_{l} F^{i}{ }_{j} \\
\quad=\delta_{j}^{k} F^{i}{ }_{l}-\delta_{l}^{i}(\eta \omega)\left(\mu_{i}, \mu_{i}\right)(\eta \omega)\left(\mu_{i}, \mu_{k}\right)(\eta \omega)\left(\mu_{k}, \mu_{j}\right)(\eta \omega)\left(\mu_{j}, \mu_{i}\right) F^{k}{ }_{j}
\end{aligned}
$$

and $F^{i}{ }_{j}$ generate a colour algebra under grading group $\Gamma$ and phase function $\eta \omega$. Note that $(\eta \omega)\left(\mu_{i}, \mu_{i}\right)=\omega\left(\mu_{i}, \mu_{i}\right)$ for all $i$.

Given a phase function $\eta$ satisfying $\eta\left(\mu_{i}, \mu_{i}\right)=1$ for all $i$, put

$$
\zeta_{i j}= \begin{cases}1, & i \leqslant j, \\ \eta\left(\mu_{i}, \mu_{j}\right), & i>j,\end{cases}
$$


so that

$$
W_{\lambda}[k]=\prod_{m=1}^{k-1} \eta\left(\mu_{k}, \mu_{m}\right)^{E^{m}{ }_{m}-\lambda_{m}}
$$

and $W_{\lambda}[1]=1$. Then

$$
\zeta_{i j} \zeta_{j i}^{-1}=\eta\left(\mu_{i}, \mu_{j}\right)
$$

and so, with this choice for $\zeta_{i j}$,

$$
\begin{aligned}
& F_{j}^{i} F^{k}{ }_{l}-(\eta \omega)\left(\mu_{i}, \mu_{k}\right)(\eta \omega)\left(\mu_{l}, \mu_{i}\right)(\eta \omega)\left(\mu_{k}, \mu_{j}\right)(\eta \omega)\left(\mu_{j}, \mu_{l}\right) F^{k}{ }_{l} F^{i}{ }_{j} \\
&= \delta_{j}^{k} F^{i}{ }_{l}-\delta_{l}^{i}(\eta \omega)\left(\mu_{i}, \mu_{i}\right)(\eta \omega)\left(\mu_{i}, \mu_{k}\right)(\eta \omega)\left(\mu_{k}, \mu_{j}\right)(\eta \omega)\left(\mu_{j}, \mu_{i}\right) F^{k}{ }_{j}
\end{aligned}
$$

If $\widetilde{\omega}\left(\mu_{i}, \mu_{i}\right)=\omega\left(\mu_{i}, \mu_{i}\right)$ for all $i$, then by putting $\eta=\widetilde{\omega} \omega^{-1}$, so $\eta\left(\mu_{i}, \mu_{i}\right)=1$ for all $i$, it follows that with an appropriate choice of $\zeta_{i j}$,

$$
\begin{aligned}
& F_{j}{ }_{j} F^{k}{ }_{l}-\widetilde{\omega}\left(\mu_{i}, \mu_{k}\right) \widetilde{\omega}\left(\mu_{l}, \mu_{i}\right) \widetilde{\omega}\left(\mu_{k}, \mu_{j}\right) \widetilde{\omega}\left(\mu_{j}, \mu_{l}\right) F^{k}{ }_{l} F^{i}{ }_{j} \\
& \quad=\delta_{j}^{k} F^{i}{ }_{l}-\delta_{l}^{i} \widetilde{\omega}\left(\mu_{i}, \mu_{i}\right) \widetilde{\omega}\left(\mu_{i}, \mu_{k}\right) \widetilde{\omega}\left(\mu_{k}, \mu_{j}\right) \widetilde{\omega}\left(\mu_{j}, \mu_{i}\right) F^{k}{ }_{j}
\end{aligned}
$$

so that $F^{i}{ }_{j}$ generate a Lie colour algebra with grading group $\Gamma$ and phase function $\tilde{\omega}$. Since $\omega_{0}(\alpha, \alpha)=\omega(\alpha, \alpha)$ for all $\alpha \in \Gamma$, then for an appropriate choice of $\zeta_{i j}$,

$$
W_{\lambda}[k]=\prod_{m=1}^{k-1}\left[\omega\left(\mu_{m}, \mu_{k}\right)^{E^{m_{m}}-\lambda_{m}} \omega_{0}\left(\mu_{m}, \mu_{k}\right)^{E^{m}{ }_{m}-\lambda_{m}}\right],
$$

and

$$
\begin{aligned}
F^{i}{ }_{j} F^{k}{ }_{l}-\omega_{0}\left(\mu_{i}, \mu_{k}\right) & \omega_{0}\left(\mu_{l}, \mu_{i}\right) \omega_{0}\left(\mu_{k}, \mu_{j}\right) \omega_{0}\left(\mu_{j}, \mu_{l}\right) F^{k}{ }_{l} F^{i}{ }_{j} \\
& =\delta_{j}^{k} F^{i}{ }_{l}-\delta_{l}^{i} \omega_{0}\left(\mu_{i}, \mu_{i}\right) \omega_{0}\left(\mu_{i}, \mu_{k}\right) \omega_{0}\left(\mu_{k}, \mu_{j}\right) \omega_{0}\left(\mu_{j}, \mu_{i}\right) F^{k}
\end{aligned}
$$

in particular, if $\mu_{i}$ and $\mu_{j}$ have the same parity, or if $\mu_{k}$ and $\mu_{l}$ have the same parity, then

$$
F^{i}{ }_{j} F_{l}{ }_{l}-F^{k}{ }_{l} F^{i}{ }_{j}=\delta_{j}^{k} F^{i}{ }_{l}-\delta_{l}^{i} F^{k}{ }_{j},
$$

and if $\mu_{i}$ and $\mu_{j}$ have opposite parity and $\mu_{k}$ and $\mu_{l}$ have opposite parity, then

$$
F^{i}{ }_{j} F^{k}{ }_{l}+F^{k}{ }_{l} F^{i}{ }_{j}=\delta_{j}^{k} F^{i}{ }_{l}+\delta_{l}^{i} F^{k}{ }_{j},
$$

so that $\left\{F^{i}{ }_{j}\right\}$ span a Lie superalgebra isomorphic to $g l(p \mid q)$, where $p$ is the number of even $\mu_{i}$, and $q$ is the number of odd $\mu_{i}(p+q=n)$. Note that $\left\{F^{i}{ }_{j}\right\}$ do not close on a Lie colour algebra. 
The tensor product of modules maps $\mathcal{O}_{\lambda} \times \mathcal{O}_{\kappa}$ to $\mathcal{O}_{\lambda+\kappa}$. Since $\Delta\left(E^{i}{ }_{j}\right)=E^{i}{ }_{j} \otimes$ $1+1 \otimes E^{i}{ }_{j}$, then

$$
\begin{aligned}
\Delta\left(z^{E^{i}{ }_{i}-\left(\lambda_{i}+\kappa_{i}\right)}\right) & =z^{E^{i}{ }_{i}-\lambda_{i}} \otimes z^{E^{i}{ }_{i}-\kappa_{i}} \\
\Delta\left(W_{\lambda+\kappa}[i]\right) & =W_{\lambda}[i] \otimes W_{\kappa}[i] \\
\Delta\left(F^{i}{ }_{j}\right) & =F^{i}{ }_{j} \otimes W_{\kappa}[i] W_{\kappa}[j]^{-1}+W_{\lambda}[i] W_{\lambda}[j]^{-1} \otimes F^{i}{ }_{j}
\end{aligned}
$$

on ordered pairs of modules in $\mathcal{O}_{\lambda} \times \mathcal{O}_{\kappa}$, where $F^{i}{ }_{j}$ is defined using $W_{\lambda}$ on modules in $\mathcal{O}_{\lambda}$ and $W_{\kappa}$ on modules in $\mathcal{O}_{\kappa}$.

Restricting to $\mathcal{O}_{0}$, then each nontrivial module has an integral weight basis, so that if $\left(\lambda_{1}, \ldots, \lambda_{n}\right)$ is a weight, that is, there exists a nonzero vector $w \in W$ such that $E^{i}{ }_{i} w=\lambda_{i} w$ for all $i$, then $\lambda_{i} \in \mathbb{Z}$ for all $i$, then $E^{i}{ }_{i}$ possesses only integer eigenvalues, so for arbitrary nonzero $z \in \mathbb{F}, z^{E^{i}}{ }_{i}$ is well defined. Note that the tensor product of modules defines a binary operation on $\mathcal{O}_{0}$. Note that (8.13) becomes

$$
W_{0}[k]=\prod_{m=1}^{k-1}\left[\omega\left(\mu_{m}, \mu_{k}\right)^{E^{m}} \omega_{0}\left(\mu_{m}, \mu_{k}\right)^{E_{m}^{m}}\right]
$$

and

$$
\begin{aligned}
\Delta\left(z^{E^{i}}{ }_{i}\right) & =z^{E^{i}{ }_{i}} \otimes z^{E^{i}}{ }_{i} \\
\Delta\left(W_{0}[i]\right) & =W_{0}[i] \otimes W_{0}[i] \\
\Delta\left(F^{i}{ }_{j}\right) & =F^{i}{ }_{j} \otimes W_{0}[i] W_{0}[j]^{-1}+W_{0}[i] W_{0}[j]^{-1} \otimes F^{i}{ }_{j}
\end{aligned}
$$

Since $W_{\lambda}[i]$ are defined in terms of the Lie colour algebra (and the representation), then $F^{i}{ }_{j}$ are defined in terms of the Lie colour algebra, as opposed to the work of Kleeman, which necessitated the introduction of operators from outside the algebra. Also, whereas Kleeman modified each homogeneous (with respect to the grading) subspace, the above modifies the generators of the Lie colour algebra.

\section{QUASITRIANGULARITY}

Here, colour quasitriangularity and its relation to non-graded quasitriangularity are reviewed.

A Hopf colour algebra $A$ is quasitriangular if there exists $\mathcal{R} \in(A \otimes A)_{0}$ such that

- $\mathcal{R} \Delta(x)=\Delta^{\prime}(x) \mathcal{R}$,

- $(\Delta \otimes 1)(\mathcal{R})=\mathcal{R}_{13} \mathcal{R}_{23}$,

- $(1 \otimes \Delta)(\mathcal{R})=\mathcal{R}_{13} \mathcal{R}_{12}$

- $\mathcal{R}$ is invertible. 
Some consequences are as follows:

- $\left(\Delta^{\prime} \otimes 1\right)(\mathcal{R})=\mathcal{R}_{23} \mathcal{R}_{13}$,

- $\left(1 \otimes \Delta^{\prime}\right)(\mathcal{R})=\mathcal{R}_{12} \mathcal{R}_{13}$,

- $\mathcal{R}_{12} \mathcal{R}_{13} \mathcal{R}_{23}=\mathcal{R}_{23} \mathcal{R}_{13} \mathcal{R}_{12}$ (colour Yang-Baxter equation),

- $(\varepsilon \otimes 1)(\mathcal{R})=(1 \otimes \varepsilon)(\mathcal{R})=u(1)$,

- $\mathcal{R}^{-1}=(S \otimes 1)(\mathcal{R})=\left(1 \otimes S^{-1}\right)(\mathcal{R})$,

- $(S \otimes S)(\mathcal{R})=\mathcal{R}$.

Here, $u(1)$ is as in Subsection 3 (iii), the Subsection on Colour Bialgebras: $u(1)$ is the multiplicative identity of $A$.

Since the universal enveloping algebra of a Lie colour algebra is colour cocommutative, then it is quasitriangular with $\mathcal{R}=1 \otimes 1$.

The results given in [10] (in which reduced grading groups were referred to as nondegenerate) are generalisable to the case where the grading group need only be finite. Specifically, if $(\Gamma, \omega)$ is the grading group and phase function (and $\Gamma$ is finite), then a colour algebra $A$ can be augmented to a unital associative algebra $\hat{A}$ using further generators $U_{\alpha}(\alpha \in \Gamma)$, subject to

$$
\begin{aligned}
U_{\alpha} U_{\beta} & =U_{\alpha+\beta}, \\
U_{\alpha} & =1, \alpha \in \Gamma_{0}, \\
U_{\alpha} x & =\omega(\alpha, \xi) x U_{\alpha}, x \in A_{\xi},
\end{aligned}
$$

and denoting the algebra monomorphism embedding $A$ into $\widehat{A}$ by $\iota_{\boldsymbol{A}}$. As in the case of [10], a graded $A$-module $M$ can be endowed with the structure of an $\widehat{A}$-module by defining

$$
U_{\alpha} m=\omega(\alpha, \mu) m, m \in M_{\mu}
$$

Call this the natural $\hat{A}$-module structure on $M$, and call the corresponding representation of $\widehat{A}$ the natural extension of the representation of $A$.

The algebra morphisms $\Psi_{A, B}, \Psi_{A, B}^{\prime}, \Phi_{A, B}$ and $\Phi_{A, B}^{\prime}$ are defined as in [10], and have the same properties as far as commutativity of diagrams is concerned. In the case where $A$ is a colour Hopf algebra, then $\widehat{A}$ is a nongraded Hopf algebra and the nongraded coproduct and nongraded antipode are defined as in [10].

Define $\mathcal{P}$ by

$$
\mathcal{P}=|\Gamma|^{-1}\left|\Gamma_{0}\right|^{-1} \sum_{\alpha, \beta \in \Gamma} \omega(\beta, \alpha) U_{\alpha} \underline{\otimes} U_{\beta}
$$

then $\mathcal{P}$ satisfies similar relations to those in $[10]$, that is, 
THEOREM. The following conditions are satisfied by $\mathcal{P}$.

(1) If $\Gamma$ is finite, $A$ and $B$ are colour algebras graded with respect to $\Gamma$, $M$ is a graded $A$-module, and $N$ is a graded $B$-module, then under the natural extension,

$$
\mathcal{P}(m \underline{\otimes} n)=\omega(\mu, \nu) m \underline{\otimes} n, m \in M_{\mu}, n \in N_{\nu} .
$$

(2) If $\Gamma$ is finite, and $A$ and $B$ are colour algebras, then

$$
\mathcal{P} \Phi(x \otimes z)=\Phi^{\prime}(x \otimes z) \mathcal{P}, \quad x \otimes z \in A \otimes B,
$$

so that $\mathcal{P}$ intertwines $\Phi$ and $\Phi^{\prime}$.

(3) If $\Gamma$ is finite, then

$$
\begin{aligned}
& (\underline{\Delta} \underline{\otimes})(\mathcal{P})=\mathcal{P}_{13} \mathcal{P}_{23} \in \widehat{A} \underline{\otimes} \widehat{A} \underline{\otimes} \widehat{B}, \\
& (1 \underline{\otimes} \underline{\Delta})(\mathcal{P})=\mathcal{P}_{13} \mathcal{P}_{12} \in \widehat{A} \underline{\otimes} \widehat{B} \underline{\otimes} \widehat{B} .
\end{aligned}
$$

(4) If $\Gamma$ is finite, then $\mathcal{P}$ is invertible with inverse

$$
\mathcal{P}^{-1}=|\Gamma|^{-1}\left|\Gamma_{0}\right|^{-1} \sum_{\alpha, \beta \in \Gamma} \omega(\alpha, \beta) U_{\alpha} \underline{\otimes} U_{\beta} .
$$

The proofs are similar to those given in [10].

If $A$ is quasitriangular, then $\widehat{A}$ is quasitriangular, with $R$-matrix $\underline{\mathcal{R}}$ given as in [10].

Similarly, if $\Gamma$ is finite, then given a solution $\mathcal{R}(x)$ of the colour parameterdependent Yang-Baxter equation,

$$
\underline{\mathcal{R}}(x)=\mathcal{P} \Phi(\mathcal{R}(x))=\Phi^{\prime}(\mathcal{R}(x)) \mathcal{P}
$$

is a solution of the nongraded parameter-dependent Yang-Baxter equation.

Note that the boundary condition

$$
\mathcal{R}(0)=I
$$

automatically gives us that

$$
\underline{\mathcal{R}}(0)=\mathcal{P},
$$

so that if the colour parameter-dependent $\boldsymbol{R}$-matrix satisfies the boundary condition (9.9) and the phase function is not trivial, then the nongraded parameter-dependent $R$-matrix does not satisfy that boundary condition. 


\section{0. $g l(p \mid q)$ : A New Coproduct, Hopf Structure AND $R$-Matrix}

Here, a set of operators corresponding to a general phase function on the grading group is considered, and this set of operators is used to relate Hopf colour algebras with isomorphic grading groups, but with different phase functions. This relation is shown to preserve colour quasitriangularity, where for each Hopf colour algebra, the phase function is the phase function appropriate to that Hopf colour algebra. The results are applied to colour $g l(n)$ : the correspondence with $g l(p \mid q)$ is identified, the $g l(p \mid q)$ generators and their coproducts for the Hopf colour algebra are identified, and the new coproducts for the Hopf superalgebra are found. This identifies a new coproduct, Hopf superalgebra structure, and $R$-matrix for the "complete" universal enveloping algebra for $g l(p \mid q)$.

Although $\left\{F^{i}{ }_{j}\right\}$ span a Lie superalgebra, the coproducts (8.17) and (8.19) are still coproducts corresponding to the phase group $\Gamma$ and phase function $\omega$.

The results in Section 9 can be generalised. Let $\widetilde{\omega}$ be a phase function on $\Gamma$, and let

$$
\widetilde{\Gamma}_{0}=\{\alpha \in \Gamma: \widetilde{\omega}(\alpha, \beta)=1, \forall \beta \in \Gamma\}
$$

A colour algebra $A$ with respect to $(\Gamma, \omega)$ can be augmented to a unital associative algebra $\tilde{A}$ using further generators $\widetilde{U}_{\alpha}(\alpha \in \Gamma)$ of grading zero subject to

$$
\begin{aligned}
\widetilde{U}_{\alpha} \widetilde{U}_{\beta} & =\widetilde{U}_{\alpha+\beta} \\
\widetilde{U}_{\alpha} & =1, \alpha \in \widetilde{\Gamma}_{0}, \\
\tilde{U}_{\alpha} x & =\widetilde{\omega}(\alpha, \xi) x \widetilde{U}_{\alpha}, x \in A_{\xi} .
\end{aligned}
$$

Here, $\widetilde{A}$ is graded with respect to $\left(\Gamma, \omega \widetilde{\omega}^{-1}\right)$. Denote the algebra monomorphism embedding $A$ into $\tilde{A}$ by $\tau_{A}$. A graded $A$-module $M$ can be endowed with the structure of an $\tilde{A}$-module by defining

$$
\tilde{U}_{\alpha} m=\tilde{\omega}(\alpha, \mu) m, m \in M_{\mu}
$$

Cali this the natural $\tilde{A}$-module structure on $M$, and call the corresponding representation of $\tilde{A}$ the natural extension of the representation of $A$ to a representation of $\tilde{A}$.

The algebra morphisms $\tilde{\Psi}_{A, B}, \widetilde{\Psi}_{A, B}^{\prime}, \widetilde{\Phi}_{A, B}$ and $\tilde{\Phi}_{A, B}^{\prime}$ are defined similarly to [10], in particular, $\widetilde{\Psi}_{A, B}, \widetilde{\Psi}_{A, B}^{\prime}: \widetilde{A \otimes B} \rightarrow \widetilde{A} \widetilde{\otimes} \widetilde{B}$ and $\widetilde{\Phi}_{A, B}, \widetilde{\Phi}_{A, B}^{\prime}: A \otimes B \rightarrow \widetilde{A} \widetilde{\otimes} \widetilde{B}$ are 
given by

$$
\begin{aligned}
\widetilde{\Psi}_{A, B}\left((x \otimes z) \widetilde{U}_{\alpha}\right) & =x \widetilde{U}_{\zeta+\alpha} \tilde{\otimes} z \widetilde{U}_{\alpha}, \quad z \in B_{\zeta}, \\
\widetilde{\Psi}_{A, B}^{\prime}\left(\widetilde{U}_{\alpha}(x \otimes z)\right) & =\widetilde{U}_{\alpha} x \widetilde{\otimes}_{U_{\xi+\alpha}} z, \quad x \in A_{\xi}, \\
\widetilde{\Phi}_{A, B}(x \otimes z) & =x \widetilde{U}_{\zeta} \widetilde{\otimes} z, \quad z \in B_{\zeta}, \\
\widetilde{\Phi}_{A, B}^{\prime}(x \otimes z) & =x \widetilde{\otimes}_{U_{\xi}} z, \quad x \in A_{\xi},
\end{aligned}
$$

and have the same properties as those in [10] as far as commutativity of diagrams is concerned. In the case where $A$ is a colour Hopf algebra with respect to $(\Gamma, \omega)$, then $\widetilde{A}$ is a Hopf colour algebra with respect to $\left(\Gamma, \omega \widetilde{\omega}^{-1}\right)$, with new coproduct, counit and antipode, given by

$$
\begin{gathered}
\tilde{\Delta}\left(x \widetilde{U}_{\alpha}\right)=\widetilde{\Phi}(\Delta(x))\left(\widetilde{U}_{\alpha} \tilde{\otimes}_{\alpha}\right)=\sum_{(x)} x_{(1)} \widetilde{U}_{\xi_{(2)}+\alpha} \tilde{\otimes} x_{(2)} \widetilde{U}_{\alpha}, \\
\tilde{\varepsilon}\left(x \tilde{U}_{\alpha}\right)=\varepsilon(x) \\
\tilde{S}\left(x \widetilde{U}_{\alpha}\right)=\tilde{U}_{-\xi-\alpha} S(x), \quad x \in A_{\xi} .
\end{gathered}
$$

The reverse coproduct is given by

$$
\tilde{\Delta}^{\prime}\left(x \tilde{U}_{\alpha}\right)=\tilde{\Phi}^{\prime}\left(\Delta^{\prime}(x)\right)\left(\tilde{U}_{\alpha} \tilde{\otimes}_{U_{\alpha}}\right) .
$$

If $\Gamma$ is finite, define $\tilde{\mathcal{P}}$ by

$$
\widetilde{\mathcal{P}}=|\Gamma|^{-1}\left|\widetilde{\Gamma}_{0}\right|^{-1} \sum_{\alpha, \beta \in \Gamma} \widetilde{\omega}(\beta, \alpha) \widetilde{U}_{\alpha} \widetilde{\otimes} \widetilde{U}_{\beta},
$$

then $\widetilde{\mathcal{P}}$ satisfies similar relations to those in [10], that is:

THEOREM. The following conditions are satisfied by $\tilde{\mathcal{P}}$.

(1) If $\Gamma$ is finite, $A$ and $B$ are colour algebras graded with respect to $(\Gamma, \omega)$, $M$ is a graded $A$-module, and $N$ is a graded $B$-module, then under the natural extension,

$$
\tilde{\mathcal{P}}(m \tilde{\otimes} n)=\widetilde{\omega}(\mu, \nu) m \tilde{\otimes} n, m \in M_{\mu}, n \in N_{\nu} .
$$

(2) If $\Gamma$ is finite, and $A$ and $B$ are colour algebras graded with respect to $(\Gamma, \omega)$, then

$$
\widetilde{\mathcal{P}} \widetilde{\Phi}(x \otimes z)=\widetilde{\Phi}^{\prime}(x \otimes z) \widetilde{\mathcal{P}}, \quad x \otimes z \in A \otimes B,
$$


so that $\tilde{\mathcal{P}}$ intertwines $\widetilde{\Phi}$ and $\widetilde{\Phi}^{\prime}$.

(3) If $\Gamma$ is finite, then

$$
\begin{aligned}
& (\widetilde{\Delta} \widetilde{\otimes} 1)(\tilde{\mathcal{P}})=\widetilde{\mathcal{P}}_{13} \widetilde{\mathcal{P}}_{23} \in \tilde{A} \tilde{\otimes} \widetilde{A} \widetilde{\otimes} \widetilde{B} \\
& (1 \widetilde{\otimes} \widetilde{\Delta})(\tilde{\mathcal{P}})=\widetilde{\mathcal{P}}_{13} \tilde{\mathcal{P}}_{12} \in \tilde{A} \widetilde{\otimes} \tilde{B} \tilde{\otimes} \widetilde{B}
\end{aligned}
$$

(4) If $\Gamma$ is finite, then $\tilde{\mathcal{P}}$ is invertible with inverse

$$
\widetilde{\mathcal{P}}^{-1}=|\Gamma|^{-1}\left|\widetilde{\Gamma}_{0}\right|^{-1} \sum_{\alpha, \beta \in \Gamma} \tilde{\omega}(\alpha, \beta) \widetilde{U}_{\alpha} \tilde{\otimes}_{\hat{Q}}
$$

The proofs are similar to those given in [10].

If $A$ is quasitriangular, then $\tilde{A}$ is quasitriangular (as a Hopf colour algebra with grading group and function $\left(\Gamma, \omega \widetilde{\omega}^{-1}\right)$ ), with $R$-matrix,

$$
\widetilde{\mathcal{R}}=\widetilde{\mathcal{P}} \widetilde{\Phi}(\mathcal{R})=\widetilde{\Phi}^{\prime}(\mathcal{R}) \widetilde{\mathcal{P}}
$$

Similarly, if $\Gamma$ is finite, and $\mathcal{R}(x)$ is a solution of the colour parameter-dependent Yang-Baxter equation, then

$$
\tilde{\mathcal{R}}(x)=\tilde{\mathcal{P}} \widetilde{\Phi}(\mathcal{R}(x))=\tilde{\Phi}^{\prime}(\mathcal{R}(x)) \tilde{\mathcal{P}}
$$

is a solution of the colour parameter-dependent Yang-Baxter equation with respect to $\left(\Gamma, \omega \tilde{\omega}^{-1}\right)$.

The boundary condition

$$
\mathcal{R}(0)=I
$$

becomes in the new algebra

$$
\tilde{\mathcal{R}}(0)=\tilde{\mathcal{P}}
$$

so that if the colour parameter-dependent $R$-matrix for $A$ satisfies the boundary condition (10.14) and the phase function $\widetilde{\omega}$ is not trivial, then the colour parameterdependent $R$-matrix for $\tilde{A}$ does not satisfy that boundary condition.

Let $\tilde{\omega}=\omega \omega_{0}=\eta^{-1}$, where $\omega_{0}$ and $\eta$ are defined as in Section 2, specifically, (2.9) and (2.12), then $\omega \tilde{\omega}^{-1}=\omega_{0}$, so that

$$
\left(\omega \widetilde{\omega}^{-1}\right)(\alpha, \beta)=\omega_{0}(\alpha, \beta)=(-1)^{\psi(\alpha) \psi(\beta)} .
$$


It follows that $\widetilde{A}$ is a Hopf superalgebra, and that all even elements of $A$ are even elements of $\widetilde{A}$, and all odd elements of $A$ are odd elements of $\widetilde{A}$.

By (8.17), the coproduct on ordered pairs of modules in $\mathcal{O}_{\lambda} \times \mathcal{O}_{\kappa}$ is

$$
\begin{aligned}
\Delta\left(z^{E^{i}{ }_{i}-\left(\lambda_{i}+\kappa_{i}\right)}\right) & =z^{E^{i}{ }_{i}-\lambda_{i}} \otimes z^{E^{i}{ }_{i}-\kappa_{i}} \\
\Delta\left(W_{\lambda+\kappa}[i]\right) & =W_{\lambda}[i] \otimes W_{\kappa}[i] \\
\Delta\left(F^{i}{ }_{j}\right) & =F^{i}{ }_{j} \otimes W_{\kappa}[i] W_{\kappa}[j]^{-1}+W_{\lambda}[i] W_{\lambda}[j]^{-1} \otimes F^{i}{ }_{j},
\end{aligned}
$$

so that in $\left[U\left(g l\left(\widetilde{\mu_{1}, \ldots, \mu_{n}}\right)\right)\right]$,

$$
\begin{aligned}
\tilde{\Delta}\left(z^{E^{i}{ }_{i}-\left(\lambda_{i}+\kappa_{i}\right)}\right) & =z^{E^{i}{ }_{i}-\lambda_{i}} \tilde{\otimes} z^{E^{i}{ }_{i}-\kappa_{i}} \\
\widetilde{\Delta}\left(W_{\lambda+\kappa}[i]\right) & =W_{\lambda}[i] \widetilde{\otimes} W_{\kappa}[i] \\
\widetilde{\Delta}\left(F^{i}{ }_{j}\right) & =F^{i}{ }_{j} \widetilde{\otimes} W_{\kappa}[i] W_{\kappa}[j]^{-1}+W_{\lambda}[i] W_{\lambda}[j]^{-1} \widetilde{U}_{\mu_{i}-\mu_{j}} \widetilde{\otimes} F^{i}{ }_{j},
\end{aligned}
$$

and on an ordered pair of modules in $\mathcal{O}_{0} \times \mathcal{O}_{0}$, the coproduct is

$$
\begin{aligned}
\tilde{\Delta}\left(z^{E^{i}{ }_{i}-\left(\lambda_{i}+\kappa_{i}\right)}\right) & =z^{E^{i}{ }_{i}-\lambda_{i}} \tilde{\otimes} z^{E^{i}{ }_{i}-\kappa_{i}} \\
\widetilde{\Delta}\left(W_{0}[i]\right) & =W_{0}[i] \tilde{\otimes} W_{0}[i] \\
\widetilde{\Delta}\left(F^{i}{ }_{j}\right) & =F^{i}{ }_{j} \widetilde{\otimes} W_{0}[i] W_{0}[j]^{-1}+W_{0}[i] W_{0}[j]^{-1} \widetilde{U}_{\mu_{i}-\mu_{j}} \tilde{\otimes} F^{i}{ }_{j} .
\end{aligned}
$$

Note that this coproduct for the Hopf superalgebra is distinct from the standard coproduct for the Hopf superalgebra $U(g l(p \mid q))$, which is given by

$$
\Delta^{s t}\left({F^{i}}_{j}\right)=F^{i}{ }_{j} \widetilde{\otimes} 1+1 \widetilde{\otimes} F^{i}{ }_{j} .
$$

Not only are (10.18) and (10.19) distinct coproducts from (10.20), but there is no algebra homomorphism of $U(g l(p \mid q))$ which will transform (10.20) into (10.18) or (10.19). This means that (10.18) and (10.19) are entirely new coproducts.

Since the coproduct $(10.20)$ is supercocommutative, then the Hopf superalgebra is quasitriangular with $R$-matrix $\mathcal{R}=1 \widetilde{\otimes} 1$. Since the coproduct (10.18) and (10.19) is not supercocommutative, then the identity $1 \widetilde{\otimes} 1$ is not an $R$-matrix for this Hopf superalgebra. The $R$-matrix corresponding to the coproduct is

$$
\mathcal{R}=\widetilde{\mathcal{P}}=|\Gamma|^{-1}\left|\widetilde{\Gamma}_{0}\right|^{-1} \sum_{\alpha, \beta \in \Gamma} \tilde{\omega}(\beta, \alpha) \widetilde{U}_{\alpha} \tilde{\otimes} \widetilde{U}_{\beta}
$$

Note that since operators $\widetilde{U}_{\alpha}$ were introduced to define the Hopf superalgebra $\left[U\left(g l\left(\widetilde{\left.\mu_{1}, \ldots, \mu_{n}\right)}\right)\right]\right.$, then $(10.18)$ and $(10.19)$ are not coproducts on $U\left(g l\left(\mu_{1}, \ldots, \mu_{n}\right)\right)$ as a Hopf superalgebra. 
On the other hand, for a phase function $\widetilde{\omega}$ on $\Gamma$ and $\alpha \in \Gamma$, define $\widetilde{V}_{\alpha}[\lambda]$ on a module in $\mathcal{O}_{\lambda}$ by

$$
\widetilde{V}_{\alpha}[\lambda]=\prod_{i=1}^{n} \widetilde{\omega}\left(\alpha, \mu_{i}\right)^{E^{i}{ }_{i}-\lambda_{i}}
$$

Then

$$
\begin{aligned}
\tilde{V}_{\alpha}[\lambda] \tilde{V}_{\beta}[\lambda] & =\tilde{V}_{\alpha+\beta}[\lambda], \\
\tilde{V}_{\alpha}[\lambda] & =1, \quad \alpha \in \widetilde{\Gamma}_{0}, \\
\tilde{V}_{\alpha}[\lambda] x & =\widetilde{\omega}(\alpha, \xi) x \widetilde{V}_{\alpha}[\lambda], \quad x \in\left(U\left(g l\left(\mu_{1}, \ldots, \mu_{n}\right)\right)\right)_{\xi},
\end{aligned}
$$

so that $\widetilde{U}_{\alpha}=\widetilde{V}_{\alpha}[\lambda]$, which are defined in terms of $g l\left(\mu_{1}, \ldots, \mu_{n}\right)$, satisfy (10.2).

By (10.18), the coproduct on ordered pairs of modules in $\mathcal{O}_{\lambda} \times \mathcal{O}_{\kappa}$ is

$$
\begin{aligned}
\widetilde{\Delta}\left(E^{i}{ }_{j}\right) & =E^{i}{ }_{j} \widetilde{\otimes} 1+V_{\mu_{i}-\mu_{j}}[\lambda] \widetilde{\otimes} E^{i}{ }_{j}, \\
\widetilde{\Delta}\left(z^{E^{i}{ }_{i}-\left(\lambda_{i}+\kappa_{i}\right)}\right) & =z^{E^{i}{ }^{i}-\lambda_{i}} \widetilde{\otimes} z^{E^{i}{ }-\kappa_{i}}, \\
\widetilde{\Delta}\left(W_{\lambda+\kappa}[i]\right) & =W_{\lambda}[i] \widetilde{\otimes} W_{\kappa}[i], \\
\widetilde{\Delta}\left(F^{i}{ }_{j}\right) & =F^{i}{ }_{j} \widetilde{\otimes} W_{\kappa}[i] W_{\kappa}[j]^{-1}+W_{\lambda}[i] W_{\lambda}[j]^{-1} \widetilde{V}_{\mu_{i}-\mu_{j}}[\lambda] \widetilde{\otimes} F^{i}{ }_{j},
\end{aligned}
$$

and on ordered pairs of modules in $\mathcal{O}_{0} \times \mathcal{O}_{0}$, the coproduct is

$$
\begin{aligned}
\widetilde{\Delta}\left(E^{i}{ }_{j}\right) & =E^{i}{ }_{j} \tilde{\otimes} 1+V_{\mu_{i}-\mu_{j}}[0] \widetilde{\otimes} E^{i}{ }_{j}, \\
\widetilde{\Delta}\left(z^{E^{i}}{ }_{i}\right) & =z^{E^{i}}{ }_{i} \widetilde{\otimes} z^{E^{i}}{ }_{i}, \\
\widetilde{\Delta}\left(W_{0}[i]\right) & =W_{0}[i] \widetilde{\otimes} W_{0}[i], \\
\widetilde{\Delta}\left({F^{i}}^{i}\right) & =F^{i}{ }_{j} \tilde{\otimes} W_{0}[i] W_{0}[j]^{-1}+W_{0}[i] W_{0}[j]^{-1} \widetilde{V}_{\mu_{i}-\mu_{j}}[0] \widetilde{\otimes} F^{i}{ }_{j} .
\end{aligned}
$$

Note that this is genuinely a coproduct for the Hopf superalgebra $U\left(g l\left(\mu_{1}, \ldots, \mu_{n}\right)\right)$ (and equivalently $U(g l(p \mid q))$ ), and is distinct from the standard coproduct (10.20).

\section{Concluding Remarks}

It is interesting that there exist different Hopf structures for the Lie superalgebra $g l(m \mid n)$, which can be obtained as a consequence of their relationship with Lie colour algebras of general linear type. Preliminary investigations suggest this phenomenon extends to other Lie superalgebras (those of types $A-G$ in Kac's notation $[5,6]$ ). More importantly, the phenomenon does extend to quantised Lie superalgebras, opening the way for the construction of more interesting solutions of the graded and ungraded Yang-Baxter equation, which may be of importance in applications. These extensions will be pursued in subsequent articles. 


\section{REFERENCES}

[1] R.J. Baxter, Exactly solvable models in statistical mechanics (Academic Press, London, 1982).

[2] H.S. Green, 'Colour algebras and generalized statistics', in Lecture Notes in Physics, Proc. XIth Int. Coll. Group Theoretical Methods in Physics 180 (Springer-Verlag, Berlin, Heidelberg, New York, 1982), pp. 346-350.

[3] H.S. Green, 'Quantization of fields in accordance with modular statistics', Austral. J. Phys. 28 (1975), 115-125.

[4] P.D. Jarvis and H.S. Green, 'Casimir invariants, characteristic identities and Young diagrams for color algebras and superalgebras', J. Math. Phys. 24 (1983), 1681-1687.

[5] V.G. Kac, 'Lie superalgebras', Adv. Math. 26 (1977), 8-96.

[6] V.G. Kac, 'Representations of classical Lie superalgebras', in Lecture Notes in Mathematics 676, Differential geometrical methods in mathematical physics II: Proceedings, University of Bonn, July 13-16, 1977 (Springer-Verlag, Berlin, Heidelberg, New York, 1978), pp. 597-626.

[7] R. Kleeman, 'Aspects of modular quantization', J. Math. Phys. 24 (1983), 166-172.

[8] R. Kleeman, 'Commutation factors on generalized Lie algebras', J. Math. Phys. 26 (1985), 2405-2412.

[9] R. Kleeman, Generalized quantization and colour algebras, Ph.D. Thesis (University of Adelaide), 1985.

[10] D.S. McAnally, 'Bosonic extension of quasitriangular colour Hopf algebras', (Univ. of Qld. preprint, 1995).

[11] K.R. Parthasarthy, Multipliers on locally compact groups, Lecture Notes in Mathematics 93 (Springer-Verlag, Berlin, Heidelberg, New York, 1969).

[12] V. Rittenberg and D. Wyler, 'Generalized superalgebras', Nuclear Phys. B 139 (1978), 189-202.

[13] M. Scheunert, 'Generalized Lie algebras', J. Math. Phys. 20 (1979), 712-720.

[14] M. Scheunert, 'Graded tensor calculus', J. Math. Phys. 24 (1983), 2658-2670.

[15] M. Scheunert, 'Casimir elements of $\varepsilon$ Lie algebras', J. Math. Phys. 24 (1983), 2671-2680.

[16] M.E. Sweedler, Hopf algebras, Notes from a course given in the Spring of 1968 at Cornell University (W.A. Benjamin, New York, 1969).

Department of Mathematics

The University of Queensland

Queensland 4072

Australia 